\title{
Imaging with SPADs and DMDs: Seeing through Diffraction-Photons
}

\author{
Ibrahim Alsolami and Wolfgang Heidrich, Senior Member, IEEE
}

\begin{abstract}
This paper addresses the problem of imaging in the presence of diffraction-photons. Diffraction-photons arise from the low contrast ratio of DMDs $(\sim 1000: 1)$, and very much degrade the quality of images captured by SPAD-based systems.

Herein, a joint illumination-deconvolution scheme is designed to overcome diffraction-photons, enabling the acquisition of intensity and depth images. Additionally, a proof-of-concept experiment is conducted to demonstrate the viability of the designed scheme. It is shown that by co-designing the illumination and deconvolution phases of imaging, one can substantially overcome diffraction-photons.
\end{abstract}

Index Terms-Time-of-Flight, SPAD, DMD, Computational Imaging.

\section{INTRODUCTION}

Today, imaging with single-photon avalanche diodes (SPADs) is rapidly gaining attention. The high sensitivity and temporal resolution of SPADs is finding favor in a variety of applications, ranging from light-in-flight recording [1] to imaging around corners [2].

Traditionally, photon detection has relied on photomultiplier tubes (PMTs). PMTs have a fast response time and provide a high amplification gain, yet they are fairly large vacuum tubes and thus not well-suited for cameras.

Nowadays, SPADs are increasingly becoming the preferred solid-state device for photon detection. Low dark counts $(<25$ $\mathrm{Hz}$ ) and high quantum efficiencies (up to $\sim 50 \%$ ) [3] are among the attractive features of SPADs.

SPAD cameras are, however, still in their infancy. Currently, commercially available SPAD cameras have low pixel resolutions $(\sim 32 \times 64$ pixels $)$. To increase the spatial resolution of single-pixel SPAD cameras, raster-scanning, whereby pixel values of a scene are sequentially acquired, is typically used.

Raster-scanning is commonly achieved by means of galvanometric mirrors. In this approach, light is steered to $x / y$ positions of a scene mechanically via a pair of mirrors. Such mechanical systems, however, are cumbersome.

In contrast to single-point illumination in galvanometric systems, digital micro-mirror devices (DMDs) can project optical patterns. A DMD is a spatial light modulator (SLM), comprising a matrix of micro-mirrors integrated with a semiconductor chip. When light shines on a DMD, high-resolution $(912 \times 1140$ pixels $)$ [4] optical patterns are projected; in our experiment, we use these optical patterns to illuminate a scene.

A challenging drawback of DMD-based projection systems, compared with their galvanometric counterparts, is the presence of scattered light. Light diffracted by edges of DMD structures, such as mirrors, gives rise to scattered light; this light can undergo multiple internal reflections within the DMD and exit as a wide cone of light, Fig. 1. Due to scattered light, scene locations that would ideally be masked by DMD mirrors in the off-state are illuminated; this imperfection hinders the collection of quality images.

The degree to which a DMD system rejects light is quantified by the contrast ratio ${ }^{1}$. The higher the contrast ratio, the lower the impact of scattered light on image quality. We shall refer to photons originating from a DMD's scattered light as diffraction-photons, Fig. 1.

There are further challenges. For example, increasing the illumination power may not always be a feasible option for enhancing image quality, such as when imaging sensitive biological samples [5]. Moreover, it is desirable to operate an imaging system in a gateless manner in order to reduce the number of measurements.

This study aims to overcome diffraction-photons and provide an alternative to raster-scanning. Using a SPAD, a DMD, and a co-designed illumination-deconvolution scheme, we experimentally demonstrate a system capable of acquiring intensity and depth images in an environment tainted by diffraction-photons.

The contributions of this study are as follows:

- Design: we design a scheme, tailored to SPAD/DMDbased imaging systems, for capturing intensity and depth images (Sections IV and V).

- Theory: we formulate image-formation models for our proposed scheme and describe techniques for recovering images from them (Section V).

- Experiment: we experimentally demonstrate the viability of our imaging system (Section VII).

The central idea of this paper can be summarized as follows:

Project overlapping illumination blocks to overcome diffraction-photons, and let deconvolution algorithms untangle pixel values.

The remainder of this paper is organized into seven sections: In Section II, we introduce some preliminaries. In Section III, we review prior work. In Section IV, we describe a method for scene illumination, and we then present our image-formation models in Section V. In Section VI, we describe details of

\footnotetext{
${ }^{1}$ The full-on/full-off contrast ratio is defined as $C R=\frac{L_{\mathrm{on}}}{L_{\mathrm{off}}}$, where $L_{\mathrm{on}}$ is the measured luminance when all DMD mirrors are in the on-state, resulting in a fully illuminated screen, and $L_{\text {off }}$ is the measured luminance when all mirrors are in the off-state.
} 


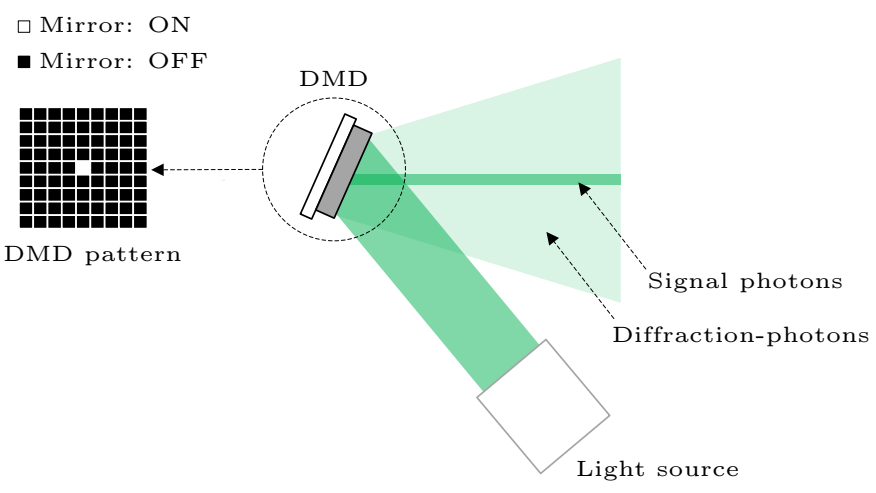

Fig. 1: Simplified sketch of diffraction-photons emanating from a DMD.

our experimental setup. In Section VII, we present and discuss our experimental results. In Section VIII, we draw conclusions from our findings and outline future research directions.

\section{Preliminaries}

In this section, we introduce some principles that will serve as starting points for the subsequent sections. This section is based in part on [6].

Each pixel, $i$, of the scene has a depth $z_{i}$ and reflectivity $\kappa_{i}$, and is illuminated $N_{r}$ times by a laser pulse with a waveform $s(t)$. The rate of photons impinging on a SPAD is given by

$$
r_{i}(t)=\kappa_{i} s\left(t-2 z_{i} / c\right)+n_{a} \quad \text { photons/sec }
$$

where $c$ is the speed of light and $n_{a}$ is the average photon rate due to ambient light.

The quantum efficiency of the SPAD lowers the photon rate by a factor of $\eta$, and dark counts, $n_{d}$, of the SPAD are added:

$$
p_{i}(t)=\eta \kappa_{i} s\left(t-2 z_{i} / c\right)+\eta n_{a}+n_{d} \quad \text { photons/sec. }
$$

A time-correlated single-photon counting (TCSPC) system quantizes the observation interval, $T_{b}$, into $m$ time-bins of duration $\Delta$. Accordingly, the average number of photon counts in a time-bin (per illumination) is

$$
\lambda_{i}^{j}=\int_{\Delta(j-1)}^{\Delta j} p_{i}(t) d t \quad \text { photons }
$$

where $j$ is the index of a time-bin, $j=1,2, \ldots, m$.

After $N_{r}$ illuminations, the number of photons, $y_{j}$, detected in a time-bin $j$ draws values from a Poisson distribution:

$$
y_{j} \sim \operatorname{Poisson}\left(N_{r} \lambda_{i}^{j}\right) \quad \text { photons. }
$$

The signal-to-background ratio (SBR), a measure of signal fidelity, is defined as follows: $\mathrm{SBR}=\frac{\lambda_{s}}{\lambda_{n}}$, where $\lambda_{s}$ and $\lambda_{n}$ are the mean number of signal and noise photons, respectively. Moreover, deadtime is defined as the time a SPAD needs to reset to its initial state after an avalanche event occurs. During this period of time, arriving photons will go undetected.

\section{PRIOR WORK}

Based on the method of acquisition employed, the literature can be categorized into the following classes:

Raster-Scanning: In raster-scanning pixel values are sequentially acquired. Typically, a pair of galvanometric mirrors or a motorized translation stage is used to obtain a scene's spatial content [7]-[10].

Our imaging system and systems in this category require the same number of measurements to reconstruct an intensity and depth image. The main problems, however, associated with mechanical scanning systems such as galvanometric mirrors or motorized translation stages is that they are substantially large, require high power levels, and in the case of galvanometric mirrors, suffer from geometric distortion [11]. A solution to these problems is to employ a DMD, which we use in our experiment.

Time-Gated Compressive Sensing: In [12]-[14], 3D imaging systems based on compressive sensing were demonstrated. Using range gating, time-slices of a scene are obtained, from which depth images are reconstructed.

While such systems can be used to reduce the impact of diffraction-photons, a full time sweep is required to construct a 3D image, as gating is needed to distinguish objects that fall within the same range interval.

What sets our imaging system apart from others in this category is that it is gateless, hence requiring fewer measurements to reconstruct a 3D image. The number of measurements in our imaging system is equal to the number of pixels, $n$, as opposed to $\left\lfloor\frac{n}{d}\right\rfloor \times m$ for gated compressed sensing, where $d$ is a selected constant for a given image sparsity/compressibility (typically in the range between 2 and 4 ) and $m$ is the number of time-bins $(m \gg d)$.

Gateless Compressive Sensing: Methods of circumventing the need for time gating have been proposed. In [15], an imaging system able to construct a $64 \times 64$ depth image in a gateless manner was demonstrated. Prior to recovering a scene's spatial information, parametric deconvolution is first used to estimate the set of depths present in scene. Once the set of depths is determined, a compressive sensing approach is used to recover a scene's spatial content.

A major obstacle to adopting the aforementioned system is the presence of deadtime-interference. To give an example, consider the following: an object in the background of a scene may not be visible as photons reflected from it may go undetected due to the deadtime initiated by preceding photons reflected from foreground objects. A way to resolve this obstacle is to use a photo diode operating in the linear-mode (analogue mode) [16], as opposed to Geiger-mode (photoncounting mode); this, however, will entail a loss in photon detection sensitivity.

In our experiment, we employ a SPAD (Geiger-mode), enabling single-photon detection sensitivity. We also use 
block illumination (Section IV), which ameliorates deadtimeinterference, allowing our imaging system to operate in gateless manner.

Epipolar Imaging: A relatively new method of image acquisition is epipolar imaging [17]-[19]. In epipolar imaging, an illumination sheet sweeps through a scene, and an array of pixels on an epipolar plane of illumination detects incoming light. This method of imaging can potentially overcome diffraction-photons as it limits indirect light. To realize such system, however, some hardware, such as controllable mirrors, is needed to select an epipolar plane. Our imaging system is free of such hardware, making it simpler to operate.

\section{ILLUMINATION}

Trading-off spatial resolution in return for a higher signal power can be made. At low illumination levels, raster-scanning (Fig. 2a) can yield inaccurate estimations of intensity and depth images due to the presence of diffraction-photons and low SBR. To improve signal power, one can scan large patches of the scene; this, however, comes at the expense of a loss in spatial resolution (Fig. 2b).

A simple yet effective approach to boosting signal power while maintaining a given spatial resolution is to use an illumination window (for example, of size $w \times w$ ) that scans the scene in steps of one pixel (Fig. 2c provides an illustration). This approach improves signal power as more photons are collected and simultaneously retains the native resolution. Additionally, deadtime-interference is alleviated as the optical power is concentrated within an illumination block, where depth values of a scene are notably correlated.

A by-product, however, of using overlapping scanning blocks (Fig. 2c) is a blur artifact as photons from adjacent pixels are collected. In Section V, we overcome this challenge via a deconvolution technique designed for the problem at hand.

A key advantage of the proposed scheme is that it provides (via optimization) a means to relax the trade-off between spatial resolution and signal power.

Let us now discuss the two prime factors that need to be taken into consideration when selecting an illumination window size, $w \times w$ :

(i) Deadtime and SPAD saturation: Increasing the window size results in more signal photons arriving at the SPAD. This, however, can improve performance only to a limited extent, because the deadtime causes the SPAD to saturate at high photon rates.

(ii) Contrast ratio and diffraction-photons: A DMD with a high contrast ratio requires a relatively small window size, because fewer diffraction-photons are emitted from it; the converse is equally true.

\section{IMAGE FORMATION}

Using the illumination scheme (Fig. 2c) presented in Section IV, we now describe approaches to recovering intensity and depth images from photon-arrival events. We first formulate the image reconstruction process as a discrete inverse problem and then solve it by a convex optimization algorithm.

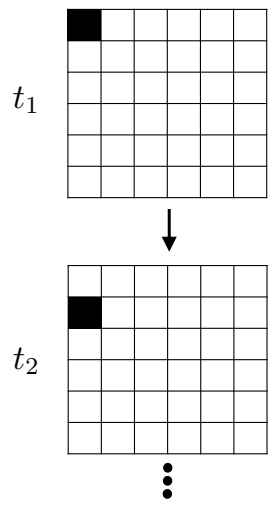

(a)

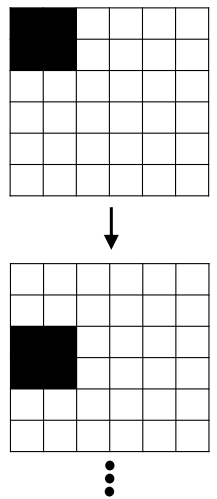

(b)

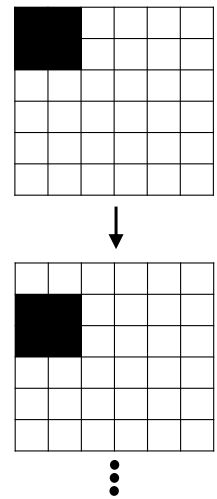

(c)
Fig. 2: (a) Raster-scanning in a pixel-by-pixel manner. (b) Raster-scanning large patches of the scene. (c) Overlap scanning. In this illustration, an illumination window of size $2 \times 2$ scans the scene in one-pixel steps.

\section{A. Intensity Image}

The goal here is to reconstruct an intensity image, $\overrightarrow{\boldsymbol{\alpha}}_{\mathrm{opt}}$, with the aid of the proposed illumination scheme (see Fig. 2c). The input-output relationship, in the absence of noise, can be described as follows:

$$
h(x, y) \circledast \alpha(x, y)=v(x, y)
$$

where $\circledast$ denotes spatial convolution, $h(x, y)$ is the pointspread function that describes the proposed illumination scheme (Fig. 2c), $\alpha(x, y)$ is the latent intensity image, and $v(x, y)$ is the observed number of photon counts at pixel $(x, y)$.

Let $\overrightarrow{\boldsymbol{\alpha}}=\left(\alpha_{1}, \ldots, \alpha_{n}\right)^{T}$ denote the vectorized representations of image $\alpha(x, y)$-column-wise stacked. Likewise, let $\overrightarrow{\boldsymbol{v}}=\left(v_{1}, \ldots, v_{n}\right)^{T}$ denote the observation vector, where $v_{i}$ is the total number of photon counts at the $i^{\text {th }}$ pixel.

The two-dimensional convolution in Eq. 5 can be expressed as matrix-vector multiplication:

$$
\begin{gathered}
\boldsymbol{H} \overrightarrow{\boldsymbol{\alpha}}=\overrightarrow{\boldsymbol{v}} \\
\left(\begin{array}{cccc}
h_{1} & h_{2} & \ldots & h_{n} \\
h_{n} & h_{1} & \ldots & h_{n-1} \\
\vdots & \vdots & \ddots & \vdots \\
h_{2} & h_{3} & \ldots & h_{1}
\end{array}\right)_{n \times n} \quad\left(\begin{array}{c}
\alpha_{1} \\
\vdots \\
\alpha_{n}
\end{array}\right)=\left(\begin{array}{c}
v_{1} \\
\vdots \\
v_{n}
\end{array}\right)
\end{gathered}
$$

where $h_{i}$ is the DMD reflection coefficient. When a pixel of the scene is within an illumination block (see Fig. 2c), $h_{i}$ takes on a value of one-full reflection. Other pixels of the scene are, however, moderately illuminated due to the low contrast ratio of the DMD: when a DMD mirror is in the off-state, a fraction of the optical power illuminates the pixels of the scene; we denote this fraction by $\epsilon$. More formally:

$h_{i}= \begin{cases}1, & \text { if } 0<i(\operatorname{MOD} \Theta) \leq w \wedge \frac{i-i(\operatorname{MOD} \Theta)}{\Theta}+1 \leq w \\ \epsilon, & \text { otherwise }\end{cases}$ where $\Theta$ denotes the number of rows of the image, and $w$ is 
the length of an illumination window of size $w \times w$ (Fig. $2 c$ provides an illustration). Additionally, let " $a$ (MOD $b$ )" denote the smallest positive integer " $q$ " such that $q \equiv a(\bmod b)$.

The following four steps are performed to recover $\overrightarrow{\boldsymbol{\alpha}}$ (Eq. 6):

\section{1) Variance-stabilizing transformation:}

In Eq. 6, $\vec{v}$ is a Poisson distributed random vector; to use an $\ell_{2}$-norm apt for Gaussian noise, we apply an Anscombe transform to convert the signal-dependent Poisson noise of $\overrightarrow{\boldsymbol{v}}$ to (approximately) additive Gaussian noise with a constant variance [20]:

$$
f\left(v_{i}\right)=2 \sqrt{v_{i}+\frac{3}{8}} .
$$

The Anscombe transformation, however, breaks the commonly used linear model expressed by Eq. 6-as $f\left(v_{i}\right)$ is nonlinear operator. We can circumvent this barrier by first denoising a blurred image (Eq. 9), then applying an inverse transform (Eq. 10), and finally recovering the latent image (Eq. 11).

\section{2) Denoising:}

The aim here is to denoise a blurred image prior to deconvolving it:

$$
\begin{gathered}
\overrightarrow{\boldsymbol{b}}_{\text {opt }}=\underset{\overrightarrow{\boldsymbol{b}}}{\operatorname{argmin}} \quad \frac{1}{2}\|\overrightarrow{\boldsymbol{b}}-f(\overrightarrow{\boldsymbol{v}})\|_{2}^{2}+\mu\|\boldsymbol{D} \overrightarrow{\boldsymbol{b}}\|_{1} \\
\text { s.t. } \quad b_{i} \geq 2 \sqrt{\frac{3}{8}} \quad \forall i, \quad i=1, \ldots, n
\end{gathered}
$$

where $\overrightarrow{\boldsymbol{b}}:=f(\boldsymbol{H} \overrightarrow{\boldsymbol{\alpha}})$ and $\overrightarrow{\boldsymbol{b}}_{\text {opt }}$ is a denoised yet blurred image. A constraint is added because the minimum value of Eq. 8 is $2 \sqrt{\frac{3}{8}}$, which occurs when $f(0)$.

\section{3) Inverse transformation:}

Algebraic inversion of Eq. 8 produces a bias; we therefore use a maximum likelihood (ML) transformation [21]:

$$
\mathcal{I}_{\mathrm{ML}}: \overrightarrow{\boldsymbol{b}}_{\mathrm{opt}} \longmapsto \overrightarrow{\boldsymbol{b}}_{*}
$$

where $\overrightarrow{\boldsymbol{b}}_{*}$ can be regarded as the denoised version of $\overrightarrow{\boldsymbol{v}}$ (Eq. 6). In this step, a lookup table is used to map the values of $\overrightarrow{\boldsymbol{b}}_{\text {opt }}$ to $\overrightarrow{\boldsymbol{b}}_{*}$.

\section{4) Deconvolution:}

We now wish to solve the following system of equations $\boldsymbol{H} \overrightarrow{\boldsymbol{\alpha}}=\overrightarrow{\boldsymbol{b}}_{*}$. Matrix $\boldsymbol{H}$ is ill-conditioned. Accordingly, we consider a regularized least-squares approach to recover our latent image:

$$
\begin{aligned}
& \overrightarrow{\boldsymbol{\alpha}}_{\mathrm{opt}}=\underset{\overrightarrow{\boldsymbol{\alpha}}}{\operatorname{argmin}} \frac{1}{2}\left\|\boldsymbol{H} \overrightarrow{\boldsymbol{\alpha}}-\overrightarrow{\boldsymbol{b}}_{*}\right\|_{2}^{2}+\lambda\|\boldsymbol{D} \overrightarrow{\boldsymbol{\alpha}}\|_{1} \\
& \text { s.t. } \quad \alpha_{i} \geq 0 \quad \forall i, \quad i=1, \ldots, n .
\end{aligned}
$$

We solve 9 and 11 using the alternating direction method of multipliers (ADMM) algorithm. Details of the ADMM algorithm are provided in the Appendix.
Limitation: A limitation of the procedure described (in steps 1-4) is that a low or high value of $\mu$ in Eq. 9 may diminish the quality of the image recovered in step 4 (Eq. 11), and thus one must carefully select $\mu$. In our experimental results (Section VII), the value of $\mu$ is chosen by inspection: the value of $\mu$ that produces the best result is selected.

\section{B. Depth Image}

The aim here is to obtain a depth image, $\vec{z}$, from photonarrival events. In the absence of noise, the input-output relationship can be expressed as follows:

$$
h(x, y) \circledast_{1} \delta(x, y, t) \circledast_{2} s(t)=r(x, y, t) .
$$

Here, two convolution processes take place:

(i) $\circledast_{1}$ is a $2 \mathrm{D}$ spatial convolution over $(x, y)$, describing the convolution of the scene with the proposed overlapping illumination blocks, $h(x, y)$ (Fig. 2c provides an example).

(ii) $\circledast_{2}$ is a $1 \mathrm{D}$ temporal convolution over $t$, describing the convolution of the scene with the waveform of the illumination pulse, $s(t)$.

Each pixel, $i$, of the scene has a depth, $z_{i}$, value and a corresponding time-of-flight $(\mathrm{ToF}), \frac{2}{c} z_{i}$, which can be represented by the following impulse response:

$$
\delta\left(t-\frac{2}{c} z_{i}\right), \quad t \in\left[0, T_{b}\right)
$$

where $\delta(\cdot)$ is a Dirac function (Fig. 3a) and $c$ is the speed of light. Likewise, in the continuous spatial and temporal domain, function $\delta(x, y, t)$ in Eq. 12 is zero except at the ToF. Additionally, let $r(x, y, t)$ denote the number of photons at a given space-time point $(x, y, t)$.

Discretization: The continuous volume containing the scene is discretized into a voxel grid: the DMD discretizes the scene spatially into $n$ pixels, and the TCSPC module discretizes the scene temporally into $m$ time-bins. In this voxel grid, a discrete depth image is represented by a vector $z \in\{1, \ldots, m\}^{n}$.

To keep the notation light, the discrete version of $\delta(x, y, t)$ in this voxel domain is denoted by $\boldsymbol{\delta}(\boldsymbol{z})$-akin to Eq. 13, but now for a complete image - and is given by

$$
\boldsymbol{\delta}(\boldsymbol{z})=\left(\begin{array}{ccc}
\delta_{0}\left(t_{1}, \frac{2}{c} z_{1}\right) & \ldots & \delta_{0}\left(t_{m}, \frac{2}{c} z_{1}\right) \\
\delta_{0}\left(t_{1}, \frac{2}{c} z_{2}\right) & \ldots & \delta_{0}\left(t_{m}, \frac{2}{c} z_{2}\right) \\
\vdots & \ddots & \vdots \\
\delta_{0}\left(t_{1}, \frac{2}{c} z_{n}\right) & \ldots & \delta_{0}\left(t_{m}, \frac{2}{c} z_{n}\right)
\end{array}\right)_{n \times m}
$$

where $t_{k} \in\{1, \ldots, m\}$ is the $k^{\text {th }}$ time-bin, $c$ is the speed of light, $z_{i}$ is the depth at pixel $i, m$ is the number of time-bins, $n$ is the total number of pixels, and $\delta_{0}(\cdot)$ is a Kronecker delta function defined as

$$
\delta_{0}(a, b)= \begin{cases}0, & \text { if } a \neq b \\ 1, & \text { if } a=b\end{cases}
$$




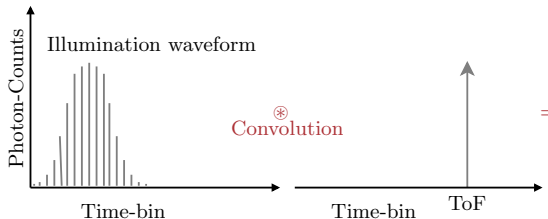

(a)

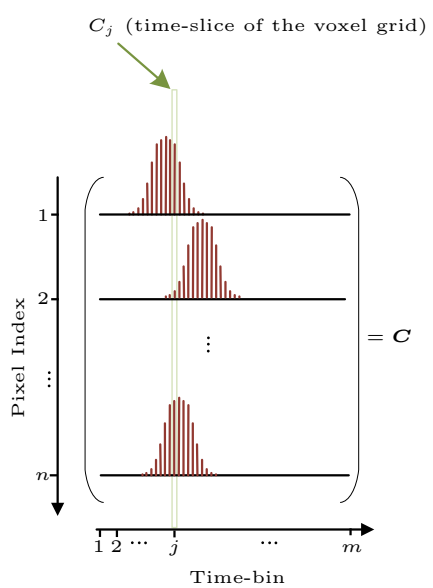

(b)

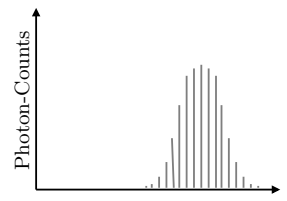

Time-bin
Fig. 3: (a) Convolution of illumination waveform with a depth point of a scene. (b) An illustrative example of the formation of $C$ and $C_{j}$. Matrix $C$ is the result of convolving the depth at each pixel with the illumination waveform. Each row of $C$ is for a given pixel, and vector $C_{j}$ is the $j^{\text {th }}$ column of $C$.

Each column of matrix $\boldsymbol{\delta}(\boldsymbol{z})$ in Eq. 14 represents a timeslice of the voxel grid at a particular time-bin, $t_{k}$, and each row is allotted to a single pixel of the scene. Additionally, matrix $\boldsymbol{\delta}(\boldsymbol{z})$ is a binary matrix, and the summation of entries along each row of $\boldsymbol{\delta}(\boldsymbol{z})$ has a value of one-as each pixel can only have a single depth.

The convolution operators in Eq. 12 can be represented as a matrix multiplication:

$$
\boldsymbol{H} \cdot \boldsymbol{\delta}(\boldsymbol{z}) \cdot \boldsymbol{S}=\boldsymbol{R}
$$

where

$$
\boldsymbol{S}=\left(\begin{array}{cccc}
s_{1} & s_{2} & \ldots & s_{m} \\
s_{m} & s_{1} & \ldots & s_{m-1} \\
\vdots & \vdots & \ddots & \vdots \\
s_{2} & s_{3} & \ldots & s_{1}
\end{array}\right)_{m \times m}
$$

Here, each row of matrix $\boldsymbol{S}$ is a time-histogram of the illumination waveform and is a circular shift of its preceding row. When a row of $\delta(\boldsymbol{z})$ is multiplied by matrix $\boldsymbol{S}$, the illumination waveform is shifted to the ToF (Fig. 3a provides a detailed example).

In Eq. 15, matrix $\boldsymbol{R}$ is the observed time-histogram at the detector (Fig. 4 provides an illustration). Each row of $\boldsymbol{R}$ is a time-histogram, resulting from convolving the scene with both an illumination window and pulse waveform:

$$
\boldsymbol{R}=\left(\begin{array}{ccc}
r_{1,1} & \cdots & r_{1, m} \\
\vdots & \ddots & \vdots \\
r_{n, 1} & \cdots & r_{n, m}
\end{array}\right)_{n \times m}
$$

Optimization: The inverse problem of recovering $z$ (Eq. 15) can be formulated using the following optimization problem:

$$
\boldsymbol{z}_{\mathrm{opt}}=\underset{\boldsymbol{z}}{\operatorname{argmin}} \frac{1}{2}\|\boldsymbol{H} \cdot \boldsymbol{\delta}(\boldsymbol{z}) \cdot \boldsymbol{S}-\boldsymbol{R}\|_{F}^{2} \quad+\quad \beta \Gamma(\boldsymbol{z})
$$

where $\Gamma(\boldsymbol{z})$ is a regularizer weighted by $\beta$. Eq. 18 is a combinatorial optimization problem, and it is challenging to solve within a reasonable period of time (the search space is $\left.m^{n}\right)$.

A tractable method for inferring depth from Eq. 18 can be attained using the following three steps:

\section{1) Spatial deconvolution:}

In Eq. 18, let $\boldsymbol{C}=\boldsymbol{\delta}(\boldsymbol{z}) \cdot \boldsymbol{S}$, and the $j^{\text {th }}$ column of $\boldsymbol{C}$ and $\boldsymbol{R}$ be denoted as $C_{j}$ and $R_{j}$, respectively (Figs. 3 and 4 provide illustrations). Both $C_{j}$ and $R_{j}$, are $n$-dimensional vectors: $C_{j}=\left(c_{1}, \ldots, c_{n}\right)^{T}$ and $R_{j}=\left(r_{1}, \ldots, r_{n}\right)^{T}$, where $j=1, \ldots, m$.

The optimization problem for each time-slice (Fig. 3b) of the voxel grid is

$$
\begin{array}{rc}
\bar{C}_{j}=\underset{C}{\operatorname{argmin}} & \frac{1}{2}\left\|\boldsymbol{H} C_{j}-R_{j}\right\|_{2}^{2}+\mu\left\|\nabla C_{j}\right\|_{1} \\
\text { s.t. } \quad c_{i} \geq 0 & \forall i, \quad i=1, \ldots, n .
\end{array}
$$

Solving this optimization problem for each $C_{j}$ yields matrix $\overline{\boldsymbol{C}}=\left(\bar{C}_{1}, \ldots, \bar{C}_{m}\right)$. We solve this convex optimization problem using an ADMM algorithm. Details of the ADMM algorithm are provided in the Appendix.

\section{2) Intermediate filtering:}

Prior to temporally deconvolving our image, we apply an $N^{\text {th }}$-order median filter. Experimentation has shown that this filter significantly improves our results.

Let $\bar{C}_{i}=\left(\bar{c}_{1}, \ldots, \bar{c}_{m}\right)$ denote the $i^{\text {th }}$ row of matrix $\bar{C}$. The $N^{\text {th }}$-order median filter of $\bar{c}_{j}$ is

$$
\hat{c}_{j}=\operatorname{median}\left(\bar{c}_{j-\left\lfloor\frac{N}{2}\right\rfloor}, \ldots, \bar{c}_{j+\left\lfloor\frac{N}{2}\right\rfloor}\right) \text {, for } j=1, \ldots, m
$$

from which we obtain $\hat{C}_{i}=\left(\hat{c}_{1}, \ldots, \hat{c}_{m}\right)$.

\section{3) Temporal deconvolution:}

Using $\hat{C}_{i}$ from the previous step, we can now determine $\vec{z}$ directly in a per-pixel manner. Vector $\hat{C}_{i}$ contains the received signal for pixel $i$, which can be regarded as a delayed and scaled version of the transmitted pulse. The ToF (or signal delay) for each pixel is independently obtained by cross-correlating $\hat{C}_{i}$ with the waveform of the transmitted pulse, $s[\cdot]$, and finding the maximum, as follows: 


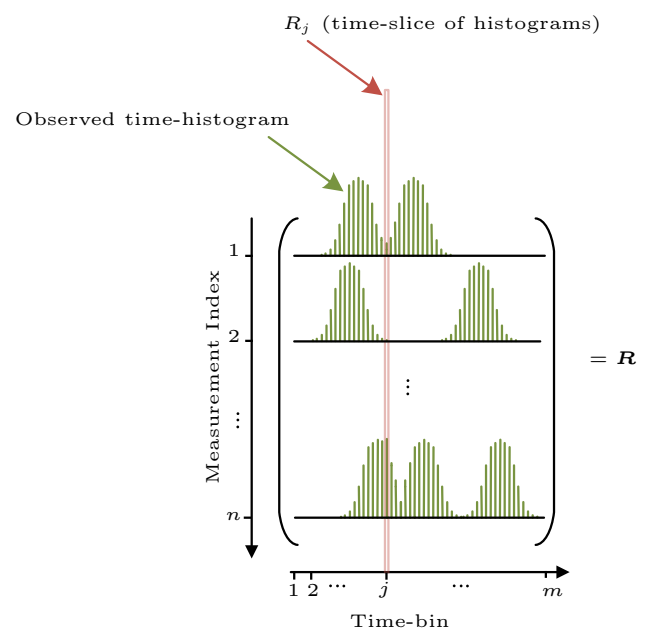

Fig. 4: An illustrative example of the formation of $\boldsymbol{R}$ and $R_{j}$. Matrix $\boldsymbol{R}$ is the observation matrix, and is the result of convolving the scene with: 1) the proposed overlapping illumination blocks 2) the illumination waveform. Each row of $\boldsymbol{R}$ is the observed time-histogram at a given measurement. Vector $R_{j}$ is the $j^{\text {th }}$ column of $\boldsymbol{R}$, and represents a time-slice of the observed histograms at time-bin $j$.

$$
t_{i}^{*}=\Delta \underset{\{0 \leq b \leq m-1\}}{\operatorname{argmax}} \sum_{a=1}^{m} \hat{C}_{i}[a] s[a-b] .
$$

For simplicity of notation, $\hat{C}_{i}[a]$ denotes the $a^{\text {th }}$ element of vector $\hat{C}_{i}$. Using Eq. 21 , the depth at pixel, $i$, is $z_{i}=$ $\frac{c}{2} t_{i}^{*}$, from which we obtain $\vec{z}=\left(z_{1}, \cdots, z_{n}\right)^{T}$.

\section{HARDWARE AND EXPERIMENTAL SETUP}

Fig. 5 shows a schematic of the experimental setup. The illumination source is a laser diode (LDH-P-C-650, PicoQuant) with a central wavelength of $\sim 656 \mathrm{~nm}$. The laser is controlled by a laser driver (PDL 828 Sepia II, PicoQuant) and emits pulses with a duration of $\sim 80$ ps (FWHM) and a repetition rate of $70 \mathrm{MHz}$.

An optical beam expander enlarges the laser beam by a factor of 5. The DMD (in a DLP 4500 projector, Texas Instruments) spatially modulates the incoming light and projects it towards the scene. The contrast ratio of the DMD is 1000:1.

Photons reflected by surfaces of the scene are detected by a SPAD (PDM $20 \mu \mathrm{m}$ Series, Micro Photon Devices) with $\mathrm{a} \sim 24$ ps $(\mathrm{FWHM})$ temporal resolution. The SPAD has a $\sim 35 \%$ quantum efficiency, $\sim 77.8 \mathrm{~ns}$ deadtime, active area diameter of $\sim 20 \mu \mathrm{m}$, and dark counts rate of $\sim 3.6 \mathrm{~Hz}$. Photons detected by the SPAD are time-stamped, relative to a sent laser pulse, with a resolution of $\sim 4$ ps by a TCSPC module (PicoHarp 300, PicoQuant).

The scene is spatially sampled by the DMD into 14440 pixels, forming an image of size 95 rows by 152 columns. The observation interval is divided by the TCSPC module into 1410 time-bins, each with a duration of $\sim 4$ ps. A total of $5 \times 10^{6}$ laser pulses are transmitted for each measurement. The integration time for each measurement is $28.2 \mathrm{~ms}$.

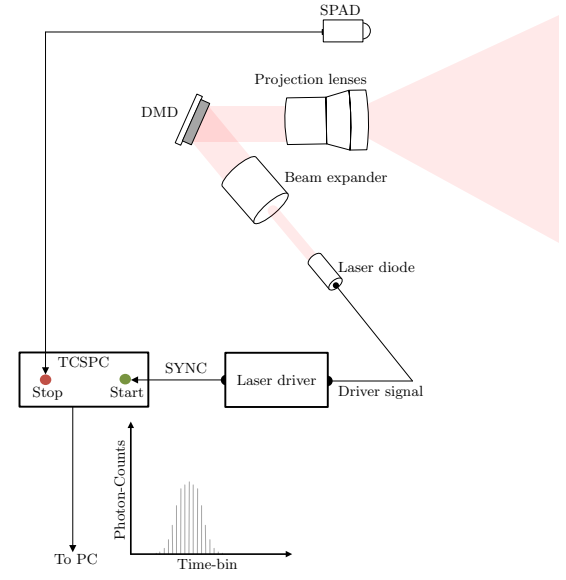

Fig. 5: Simplified schematic of experimental setup.

The scene consists of a ball ( $\sim 11 \mathrm{~cm}$ radius) placed in front of a screen. The ball is $\sim 50 \mathrm{~cm}$ away from the SPAD, and the horizontal distance between the SPAD and the background screen is $\sim 72 \mathrm{~cm}$. Table I provides a summary of the experimental parameters.

TABLE I: Experimental parameters

\begin{tabular}{lr}
\hline Parameter & Value \\
\hline \hline Average dark-count rate of SPAD & $\sim 3.6 \mathrm{~Hz}$ \\
Center wavelength of laser & $\sim 656 \mathrm{~nm}$ \\
Contrast ratio of DMD & $\sim 1000: 1$ \\
Deadtime ${ }^{\dagger}$ of SPAD & $\sim 77.8 \mathrm{~ns}$ \\
Diameter of active area of SPAD & $\sim 20 \mu \mathrm{m}$ \\
Integration time per measurement & $28.2 \mathrm{~ms}$ \\
Laser pulse duration & $\sim 80 \mathrm{ps}$ \\
Number of transmitted laser pulses per measurement & $5 \times 10^{6}$ \\
Number of time-bins & 1410 \\
Quantum efficiency of SPAD & $\sim 35 \%$ \\
Repetition rate of laser pulses & $70 \mathrm{MHz}$ \\
Timing resolution of SPAD & $\sim 24 \mathrm{ps}$ \\
Timing resolution of TCSPC module & $\sim 4 \mathrm{ps}$
\end{tabular}

${ }^{\dagger}$ Non-extensible deadtime [22]. ${ }^{\ddagger}$ Full width at half maximum.

\section{RESUlTS AND DisCUSSION}

In this section, we present and discuss the results of a proof-of-concept experiment. The goal is to demonstrate the ability of the designed illumination-deconvolution scheme (Sections IV and V) to overcome diffraction-photons.

Fig. 6 displays histograms of our experimental data, and the statistics of our measurements are as follows: the average number of noise (ambient + dark) photons was measured and found to be $0.2 \pm 0.5$ photons/pixel. The average number diffraction-photons with noise was measured and found to be $25.8 \pm 5.2$ photons/pixel. For consistency, we have taken 14440 samples for all measurements presented in this section-this value is equal to the number of pixels of the sought image.

The average number of photons (signal + noise + diffraction) per pixel for raster-scanning, and illumination blocks of size $3 \times 3,5 \times 5$, and $7 \times 7$, was measured and found to be $26.8 \pm 5.4,36.2 \pm 14.0,55.1 \pm 35.3$, and $83.7 \pm 67.1$ photons/pixel, respectively. 

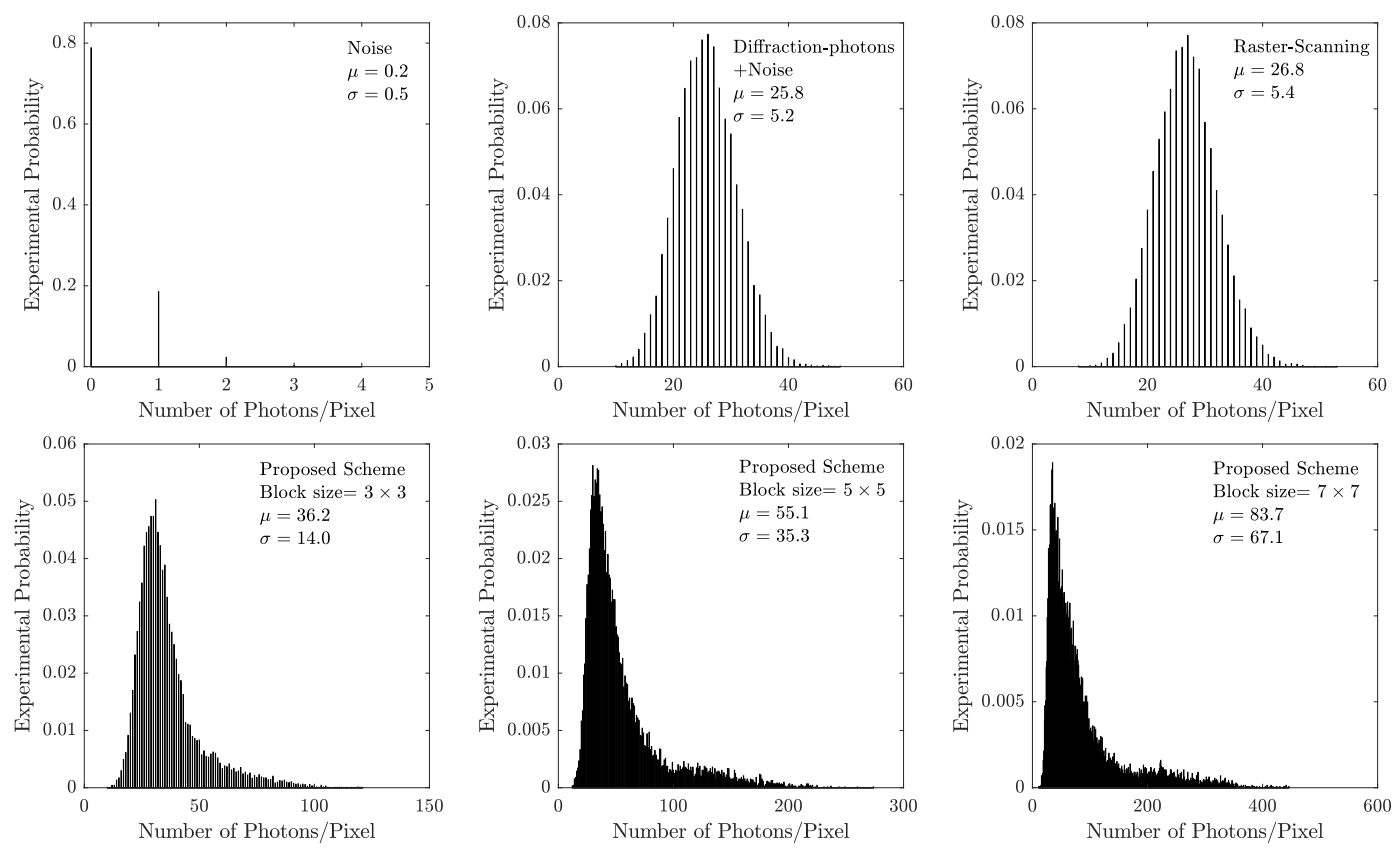

Fig. 6: Histograms of experimental data. Supplementary details: for raster-scanning and the proposed scheme, the measured photons/pixel comprise signal, noise and diffraction photons.

Fig. $7 \mathrm{~b}$ and Figs. 8a-8e, display data gathered from the experiment for the observation vector $\vec{v}$ (Eq. 6) and matrix $\boldsymbol{R}$ (Eq. 17), respectively. Fig. 7d and Figs. 8h-8j present the performance of the proposed scheme.

A rich body of research exists on 3D structure and reflectivity imaging with photon-counting detectors. However, this is the first paper to address the issue of diffraction-photons in such systems. We therefore believe that, in addition to presenting the performance of our scheme, it would be beneficial to compare it with commonly used benchmarks ([6] and [7]).

Fig. 7 and 8 show that the proposed scheme reveals a latent image amid a bath of diffraction-photons $(\sim 25$ photons/pixel, Fig. 6). Methods based on traditional raster-scanning [6], [7], however, are adversely affected by diffraction-photons. The reason for this is that the average number of signal photons per pixel for raster-scanning is greatly below the number of diffraction-photons; this results in diminished image quality.

The proposed scheme has an improved performance as it takes co-designed illumination and deconvolution approach to solve the image capturing problem, which boosts the average number of signal photons collected per pixel (Fig. 6) and maintains the native image resolution as the relatively large illumination blocks overlap (Fig. 2). For our experiment, an illumination window size of $5 \times 5$ produced the best results: this size is a workable balance between signal photons collected and blur introduced.

It is important to point out, however, that the performance improvement of the proposed scheme over [6] and [7] should be viewed with some caution, because [6] and [7] are designed for imaging environments without diffraction-photons. As such, they produce a lower image quality than our scheme.

\section{CONCLUSION}

This study set out to overcome diffraction-photons. We described a method for acquiring intensity and depth images in an environment tainted by diffraction-photons $(\sim 25$ photons/pixel).

A proof-of-concept experiment demonstrates the viability of the designed scheme. It is shown that at a low DMD contrast ratio $(\sim 1000: 1)$, diffraction-photons can be overcome via a joint illumination-deconvolution scheme, enabling the acquisition of intensity and depth images.

The scheme works as the number of signal photons collected is boosted by overlapping sizeable illumination blocks. The overlapping blocks mix pixel values, which are subsequently untangled by deconvolution algorithms.

The central conclusion that can be drawn from this study is that the designed scheme offers a means to relax the trade-off between spatial resolution and signal power-this is achieved mainly through convex optimization techniques.

A promising avenue for future research is to determine the optimal illumination window size mathematically, for a given contrast ratio, deadtime and noise level. Thus far, the optimal window size has been determined by experimental testing. Additionally, a potential research direction is to analyze the sensitivity of the problems in Eqs. 11 and 19 to noise in $\boldsymbol{H}$. Such an analysis would provide insight into how to best deconvolve images captured by SPAD/DMD-based imaging systems.

To conclude, we believe that the findings in this paper will be of special interest to researchers in the field of ToF imaging as it addresses a new practical challenge. 


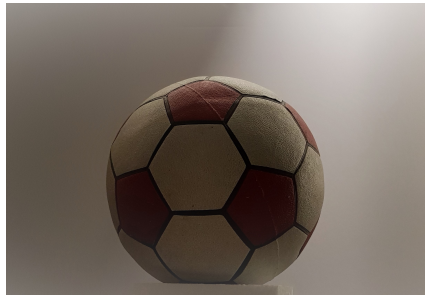

(a) Scene
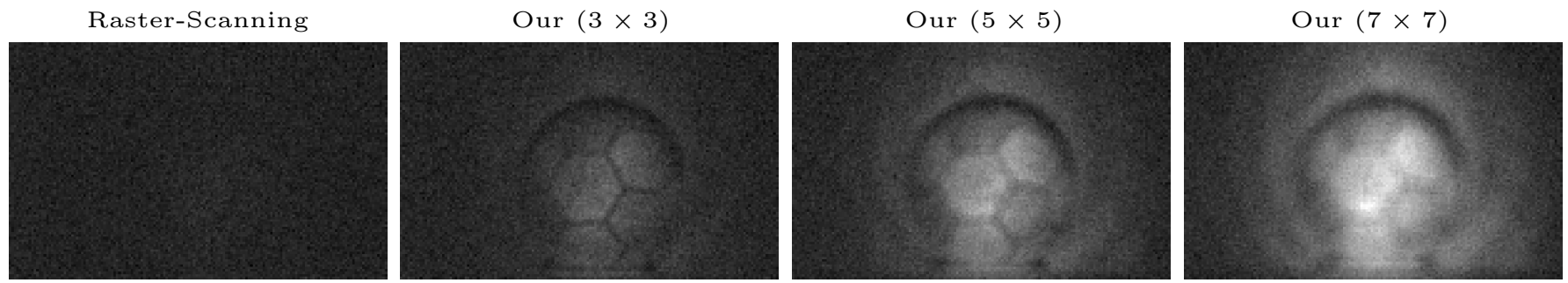

(b) Experimental data. A display of observation vectors $\vec{v}$, Eq. 6. Supplementary details: for a consistent comparison, images shown here have the same photon-count to grayscale map (i.e., a black pixel represents the minimum photon-count among all the four images. Similarly, a white pixel represents the maximum photon-count among all the four images. Photon-counts between the maximum and minimum are mapped to a gray color). For the proposed scheme, the illumination window size $(w \times w)$ is varied from $3 \times 3$ to $7 \times 7$. All images are gamma-corrected, $\gamma=2.2$.

Raster-Scanning

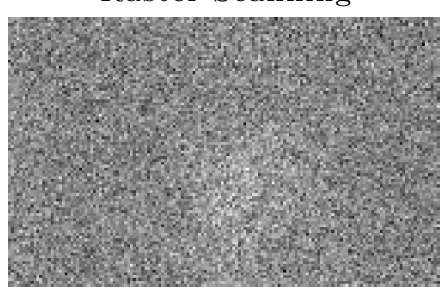

$\operatorname{Our}(3 \times 3)$

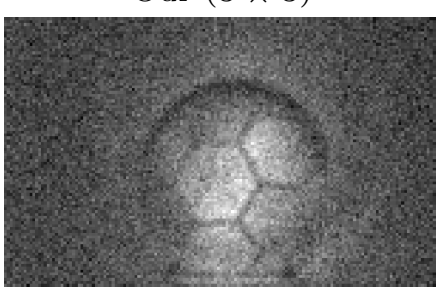

Our $(5 \times 5)$

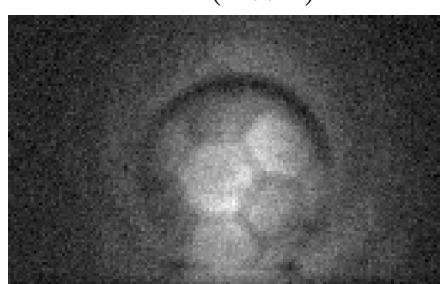

$\operatorname{Our}(7 \times 7)$

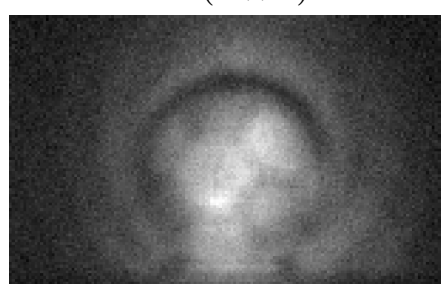

(c) Experimental data. Images of Fig. 7b with an independent grayscale map. Supplementary details: to enhance, to some extent, the visibility of the images shown in Fig. 7b, each image here has an independent grayscale map (i.e., the minimum and maximum photon-counts of each individual image is mapped to a black and white pixel, respectively. Photon-counts between the maximum and minimum are mapped to a gray color). All images are gamma-corrected, $\gamma=2.2$.

Ref. [7]

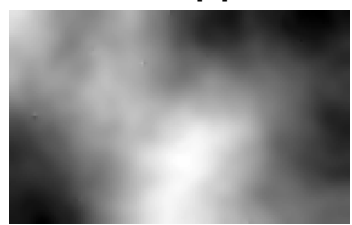

Ref. [6]

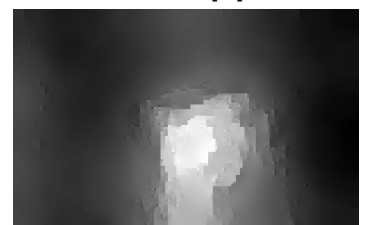

Our $(3 \times 3)$

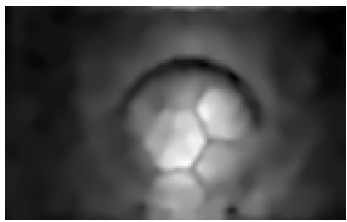

Our $(5 \times 5)$

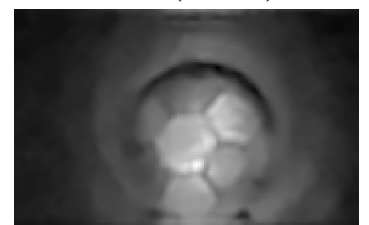

$\operatorname{Our}(7 \times 7)$

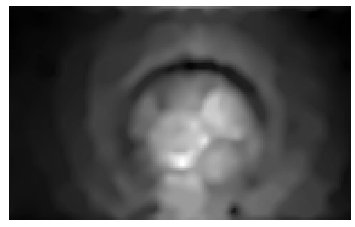

(d) The end-results. Denoised/deconvolved images. Supplementary details: the input data for both Ref. [6] and [7] is obtained from the image Raster-Scanning displayed in Fig. 7c. For Ref. [7], the input data is the number of transmitted pulses until the first photon arrives. For Ref. [6], the input noisy image is the intensity image labeled Raster-Scanning in Fig. 7c. For the proposed scheme, the input noisy/blurred images for $3 \times 3,5 \times 5$, and $7 \times 7$, are their corresponding images shown in Fig. 7c. All images here have an independent grayscale map and are gamma-corrected, $\gamma=2.2$.

Fig. 7: Intensity images. 


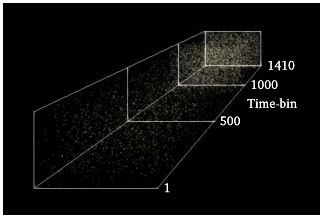

(a) First photon of raster-scanning

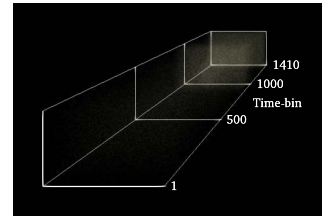

(b) All photons of raster-scanning

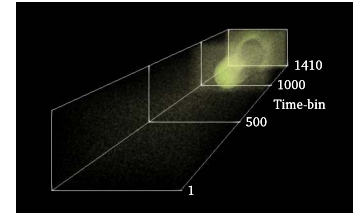

(c) Our $(3 \times 3)$

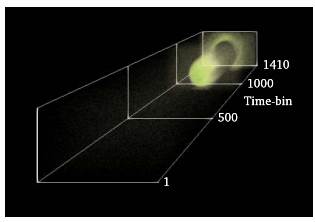

(d) Our $(5 \times 5)$

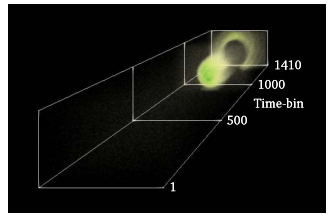

(e) Our $(7 \times 7)$

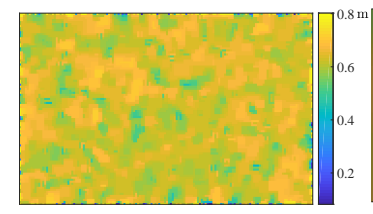

(f) Ref. [7]

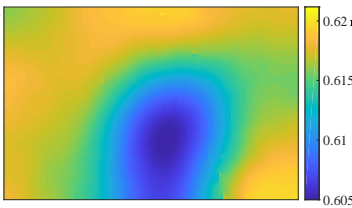

(g) Ref. [6]

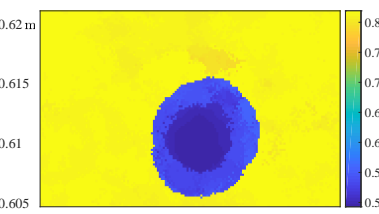

(h) Our $(3 \times 3)$

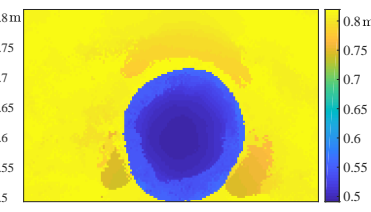

(i) Our $(5 \times 5)$

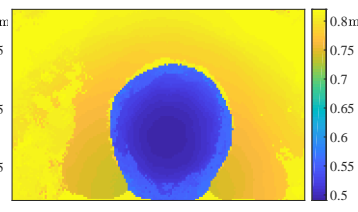

(j) Our $(7 \times 7)$

Fig. 8: (a)-(e) Raw data: a visualization of experimental data (first photon of raster-scanning and matrix $\boldsymbol{R}$, Eq. 17). (f)-(j) Depth images. Supplementary details: each column of $\boldsymbol{R}$ is reshaped to form a $95 \times 152$ image, so as to visualize $\boldsymbol{R}$ in a $3 \mathrm{D}$ space $(95 \times 152 \times 1410)$. In the experiment, we vary the illumination window size from $3 \times 3$ to $7 \times 7$ for the proposed scheme. The opacity of points in figure Fig. $8 \mathrm{a}$ are considerably higher than that of figures in the first row, so as to enhance visibility.

\section{APPENDIX A}

\section{ALTERNATING DIRECTION METHOD OF MULTIPLIERS}

In this section we shall denote the shrinkage operator (element-wise soft thresholding) by

$$
\mathcal{S}_{\tau}(x):= \begin{cases}x-\tau, & x>\tau \\ 0, & |x| \leq \tau \\ x+\tau, & x<-\tau\end{cases}
$$

Additionally, we denote the projection of $x$ onto set $\Omega=$ $[2 \sqrt{3 / 8},+\infty)$ and $\mathbb{R}_{+}$by $\operatorname{Proj}_{\Omega}(x):=\max \{x, 2 \sqrt{3 / 8}\}$ and $\operatorname{Proj}_{\mathbb{R}_{+}}(x):=\max \{x, 0\}$, respectively.

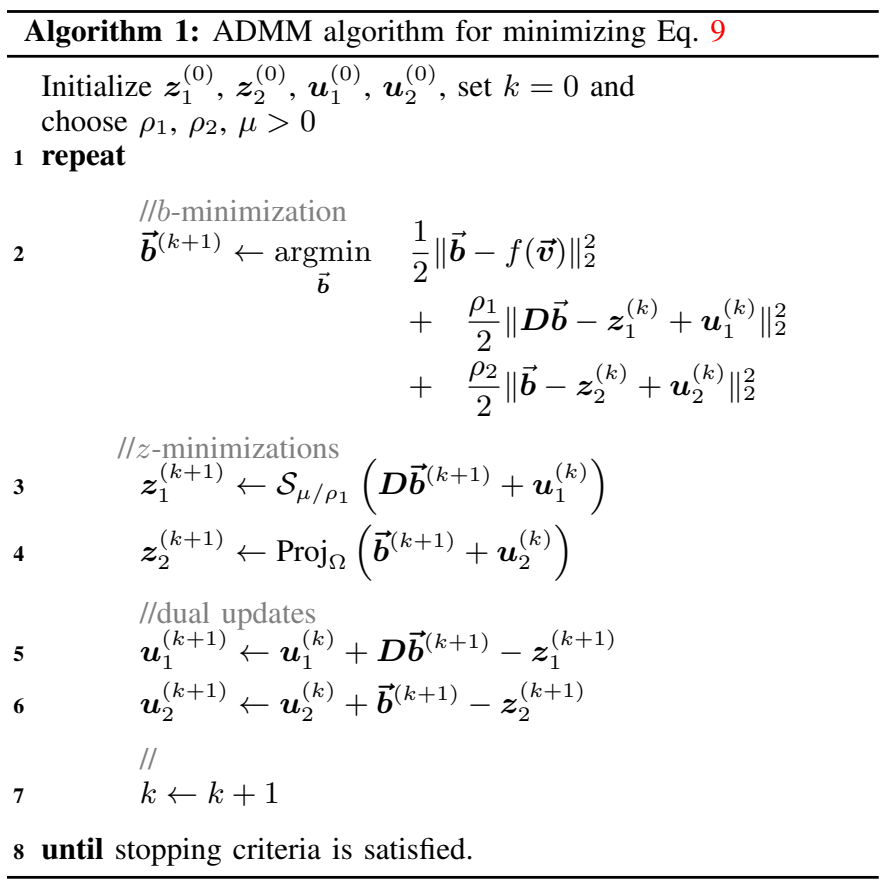

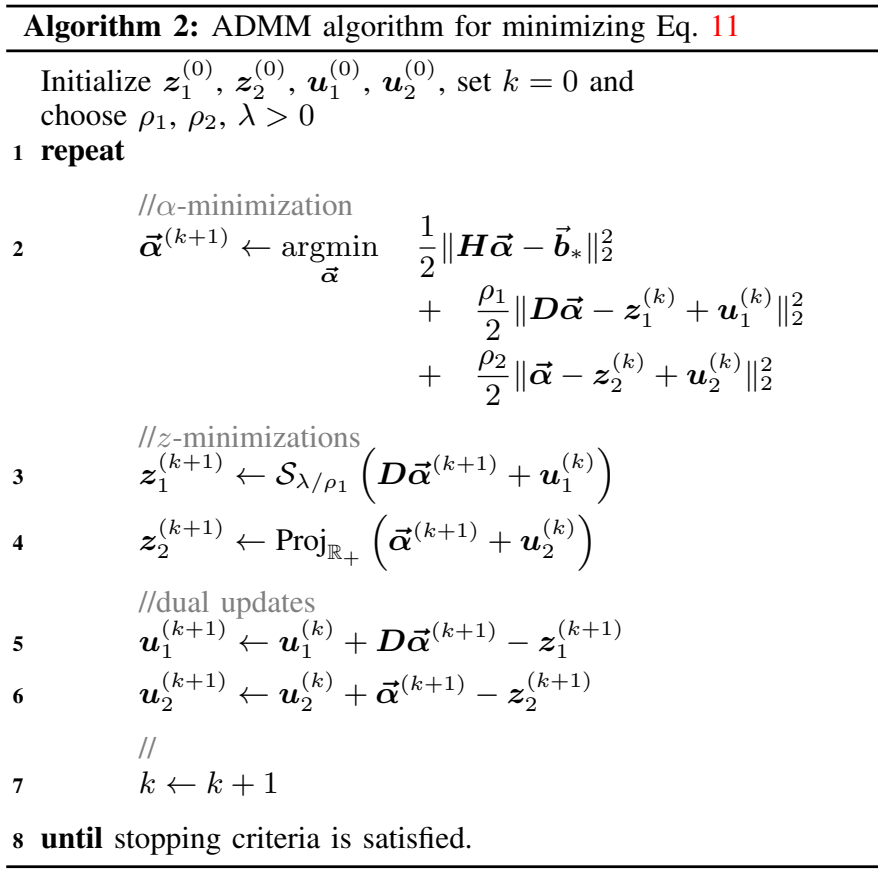

\section{APPENDIX B}

DERIVATIVE MATRICES

In this section we describe the derivative matrices used throughout the paper. Let $\boldsymbol{D} \in \mathbb{R}^{8 n \times n}$ be defined as follows:

$$
\boldsymbol{D}=\left(\begin{array}{c}
\nabla \\
\rho \nabla^{\prime \prime}
\end{array}\right)_{8 n \times n}
$$

where $\nabla$ and $\nabla^{\prime \prime}$ are the first and second order derivative matrices, respectively; and constant $\rho$ controls the strength of the second derivative. Here, the first and second derivative matrices are defined as 


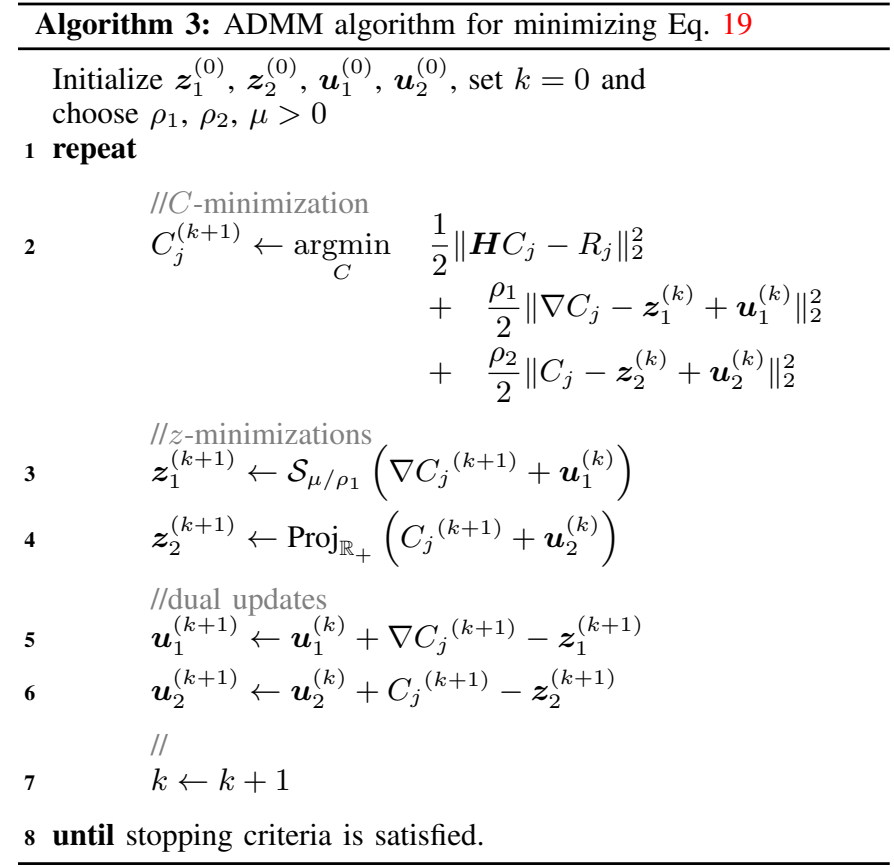

$$
\nabla=\left(\begin{array}{c}
\mathcal{A}_{x} \\
\mathcal{A}_{y} \\
\mathcal{A}_{x y} \\
\mathcal{A}_{y x}
\end{array}\right)_{4 n \times n}
$$

and

$$
\nabla^{\prime \prime}=\left(\begin{array}{c}
\mathcal{A}_{x}^{\prime \prime} \\
\mathcal{A}_{y}^{\prime \prime} \\
\mathcal{A}_{x y}^{\prime \prime} \\
\mathcal{A}_{y x}^{\prime \prime}
\end{array}\right)_{4 n \times n}
$$

where $\mathcal{A}, \mathcal{A}^{\prime \prime} \in \mathbb{R}^{n \times n}$ are convolution matrices for the first and second derivatives, respectively; and their subscripts specify the direction in which a derivative operation is performed.

The rationale of including a second derivative in our regularizer is that it encourages the recovery of image curvatures, rendering deblurred images more naturally-looking.

\section{ACKNOWLEDGMENT}

The authors wish to express their gratitude to Congli Wang, Muxingzi Li and Qilin Sun for their technical assistance and fruitful discussions. This research was made possible by KAUST Baseline Funding.

\section{REFERENCES}

[1] G. Gariepy, N. Krstajić, R. Henderson, C. Li, R. R. Thomson, G. S. Buller, B. Heshmat, R. Raskar, J. Leach, and D. Faccio, "Single-Photon Sensitive Light-in-F[1]ight Imaging," Nature communications, vol. 6, p. 6021, 2015.
[2] M. O'Toole, D. B. Lindell, and G. Wetzstein, "Confocal Non-Line-ofSight Imaging Based on the Light-Cone Transform," Nature, 2018.

[3] PicoQuant, "PDM Series - Single Photon Avalanche Diodes," Datasheet, September 2015.

[4] Texas Instruments, "DLP4500 (0.45 WXGA DMD)," DLPSO28C datasheet, April 2013 [Revised Feb. 2018].

[5] P. Hinterdorfer and A. Van Oijen, Handbook of Single-Molecule Biophysics. Springer Science \& Business Media, 2009, pp. 73.

[6] D. Shin, A. Kirmani, V. K. Goyal, and J. H. Shapiro, "Photon-Efficient Computational 3-D and Reflectivity Imaging with Single-Photon Detectors," IEEE Transactions on Computational Imaging, vol. 1, no. 2, pp. $112-125,2015$

[7] A. Kirmani, D. Venkatraman, D. Shin, A. Colaço, F. N. Wong, J. H. Shapiro, and V. K. Goyal, "First-Photon Imaging," Science, vol. 343, no. 6166, pp. 58-61, 2014.

[8] M. O'Toole, F. Heide, D. B. Lindell, K. Zang, S. Diamond, and G. Wetzstein, "Reconstructing Transient Images from Single-Photon Sensors," in Proc. IEEE CVPR, 2017, pp. 2289-2297.

[9] W. He, Z. Feng, J. Lin, S. Shen, Q. Chen, G. Gu, B. Zhou, and P. Zhang, "Adaptive Depth Imaging with Single-Photon Detectors," IEEE Photonics Journal, vol. 9, no. 2, pp. 1-12, 2017.

[10] D. Shin, F. Xu, D. Venkatraman, R. Lussana, F. Villa, F. Zappa, V. K. Goyal, F. N. Wong, and J. H. Shapiro, "Photon-Efficient Imaging with a Single-Photon Camera," Nature communications, vol. 7, p. 12046, 2016.

[11] Thorlabs, Inc, "GVS011 and GVS012 Large Mirror Diameter Scanning Galvo Systems," User Guide, HA0220T [Revised 7 July 2011].

[12] G. A. Howland, P. Zerom, R. W. Boyd, and J. C. Howell, "Compressive Sensing LIDAR for 3D Imaging," in Lasers and Electro-Optics (CLEO), 2011 Conference on. IEEE, 2011, pp. 1-2.

[13] L. Li, L. Wu, X. Wang, and E. Dang, "Gated Viewing Laser Imaging with Compressive Sensing," Applied optics, vol. 51, no. 14, pp. 27062712, 2012.

[14] L. Li, W. Xiao, and W. Jian, "Three-Dimensional Imaging Reconstruction Algorithm of Gated-Viewing Laser Imaging with Compressive Sensing," Applied optics, vol. 53, no. 33, pp. 7992-7997, 2014.

[15] A. Colaço, A. Kirmani, G. A. Howland, J. C. Howell, and V. K. Goyal, "Compressive Depth map Acquisition using a Single Photon-Counting Detector: Parametric Signal Processing meets Sparsity," in Computer Vision and Pattern Recognition (CVPR), 2012 IEEE Conference on. IEEE, 2012, pp. 96-102.

[16] M.-J. Sun, M. P. Edgar, G. M. Gibson, B. Sun, N. Radwell, R. Lamb, and M. J. Padgett, "Single-Pixel Three-Dimensional Imaging with TimeBased Depth Resolution," Nature communications, vol. 7, p. 12010, 2016.

[17] S. Achar, J. R. Bartels, W. L. Whittaker, K. N. Kutulakos, and S. G. Narasimhan, "Epipolar Time-of-Flight Imaging," ACM Transactions on Graphics (ToG), vol. 36, no. 4, p. 37, 2017.

[18] M. O'Toole, S. Achar, S. G. Narasimhan, and K. N. Kutulakos, "Homogeneous Codes for Energy-Efficient Illumination and Imaging," $A C M$ Transactions on Graphics (ToG), vol. 34, no. 4, p. 35, 2015.

[19] M. O'Toole, J. Mather, and K. N. Kutulakos, "3D Shape and Indirect Appearance by Structured Light Transport," in Computer Vision and Pattern Recognition (CVPR), 2014 IEEE Conference on. IEEE, 2014, pp. 3246-3253.

[20] F. J. Anscombe, "The Transformation of Poisson, Binomial and Negative-Binomial Data," Biometrika, vol. 35, no. 3/4, pp. 246-254, 1948.

[21] M. Mäkitalo and A. Foi, "Optimal Inversion of the Anscombe Transformation in Low-Count Poisson Image Denoising," IEEE transactions on Image Processing, vol. 20, no. 1, pp. 99-109, 2011.

[22] M. L. Larsen and A. B. Kostinski, "Simple Dead-Time Corrections for Discrete Time Series of Non-Poisson Data," Measurement Science and Technology, vol. 20, no. 9, p. 095101, 2009.

[23] Z. T. Harmany, R. F. Marcia, and R. M. Willett, "This is SPIRALTAP: Sparse Poisson Intensity Reconstruction Algorithms-Theory and Practice," IEEE Transactions on Image Processing, vol. 21, no. 3, pp. 1084-1096, 2012 (Note: the MATLAB codes of Ref. [6] and [7] are based on the code of this journal. We therefore include this journal in our reference list). 